\title{
Semi-synthetic analogs of 17-hydroxycativic acid and their biological evaluation
}

\author{
Natalia Paola Alza ${ }^{1}$ and Ana Paula M urray ${ }^{1}$ \\ ${ }^{1}$ INQUISUR-CONICET, Departamento de Química, Universidad Nacional del Sur, Avenida \\ Alem 1253, Bahía Blanca, 8000, Argentina. natalia.alza@uns.edu.ar
}

\begin{abstract}
A new series of analogs of the naturally occurring 17-hydroxycativic acid (1) were synthesized and evaluated as cholinesterase inhibitors. All derivatives have been fully characterized by mono- and bidimensional NMR spectroscopy. The butyrylcholinesterase (BChE) inhibitory activity of the analogs was of the same order of magnitude as that of compound $\mathbf{1}\left(\mathrm{IC}_{50}=171.1 \mu \mathrm{M}\right)$, while the acetylcholinesterase (AChE) inhibition was not improved by these derivatizations, being $\mathbf{1}$ the most potent $A C h E$ inhibitor of the series $\left(I C_{50}=21.1 \mu \mathrm{M}\right)$. These results suggest that both carboxylic group (C15) and allylic alcohol (C17) could be important for AChE inhibitory activity.
\end{abstract}

\section{Keywords}

Grindelia ventanensis, cholinesterase inhibition, 17-hydroxycativic acid, semisynthesis.

\section{Introduction}

Alzheimer's disease (AD) is a progressive neurodegenerative disorder associated with memory impairment and cognitive deficit. Although many factors have been implicated in $A D$, its etiology and pathogenesis remains unclear. The cholinergic hypothesis postulates that at least some of the cognitive decline results from the low levels of the neurotransmitter acetylcholine (ACh) in the brain of $A D$ patients. The inhibition of acetylcholinesterase (AChE), the enzyme that catalyzes $A C h$ hydrolysis, is the main therapeutic strategy followed to treat $A D$. Recent reports suggest that drug development would be targeted not only to increasing AChE inhibition but also selectivity for AChE over butyrylcholinesterase (BuChE) ${ }^{1}$, which activity decreases in synapses of AD patients.

Labdane diterpenes have demonstrated several biological activities, such as antiproliferative against different cancer cell lines and gastroprotective ${ }^{2-6}$. Structural modifications of these diterpenes had led derivatives with improved bioactivities ${ }^{3-6}$.

In the course of our ongoing studies of natural products from our regional flora with AChE inhibitory activity, a bioassay-guided fractionation of the ethanolic extract of Grindelia ventanesis Bartola \& Tortosa (Asteraceae) resulted in the isolation of a labdane diterpene of the normal series identified as 17-hydroxycativic acid (1). Taking into account that $\mathbf{1}$ showed a significant inhibition of $A C h E\left(I C_{50}=21.1 \mu \mathrm{M}\right)$, selectivity over BuChE $\left(I C_{50}=171.1 \mu \mathrm{M}\right)$ and that it was easily isolated from the plant extract in a very good yield ( $150 \mathrm{mg} / 100 \mathrm{~g}$ of aerial parts), we decided to obtain semisynthetic analogs of this natural diterpene through simple structural modifications of two of the three hot spots of this molecule: the carboxylic group (C15) and the primary allylic alcohol (C17) (Figure 1). 


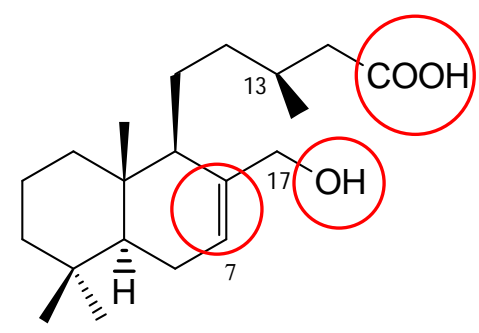

Figure 1 Hot spots of compound $\mathbf{1}$.

\section{Experimental}

General. NMR measurements were carried out at $22{ }^{\circ} \mathrm{C}$ from DMSO- $\mathrm{d}_{6}$ or $\mathrm{CDCl}_{3}$ solutions, on a Bruker ARX300 spectrometer operated at 300 and $75 \mathrm{M} \mathrm{Hz}$ for hydrogen and carbon, respectively. Chemical shifts are given in ppm $(\delta)$ with TMS as an internal standard. Silica gel 60 (Merck, 200 425 mesh) was used for column chromatography. Analytical TLC was performed on Silicagel $60 \mathrm{~F}_{254}$ sheets $(0.2 \mathrm{~mm}$ thickness, Merck). The $p$-anisaldehyde-acetic acid spray reagent was used for detection. UV spectra were recorded on a JASCO V-630BIO spectrophotometer. Microwave assisted reactions were carried out in a CEM Discover reactor. Acetylcholinesterase from electric eel (type VI-S), 5,5'-Dithiobis(2-nitrobenzoic acid) (DTNB), acetylthiocholine iodide (ATCI), butyrylthiocholine iodide (BTCl) and tacrine were purchased from Sigma. Butyrylcholinesterase (horse serum) was purchased from M P Biomedicals.

Synthesis of 2. $\left(\mathrm{CH}_{3}\right)_{3} \mathrm{~N} \cdot \mathrm{SO}_{3}(0.4 \mathrm{mmol})$ was added to a solution of $\mathbf{1}(0.1 \mathrm{mmol})$ in dry DMF $(3 \mathrm{ml})$. The reaction mixture was irradiated and stirred at $150^{\circ} \mathrm{C}$ for $5 \mathrm{~min}$ in a sealed tube in a microwave reactor. After evaporation to dryness, the residue was eluted through Amberlite CG-120 (sodium form) with methanol $(50 \mathrm{ml})$, evaporated under reduced pressure and purified by column chromatography on silicagel using $\mathrm{CH}_{2} \mathrm{Cl}_{2} / \mathrm{MeOH}$. Fractions eluted with $\mathrm{CH}_{2} \mathrm{Cl}_{2} / \mathrm{MeOH}$ (87.5:12.5) afforded pure 2 (23.7 mg, $55 \%$ ). For ${ }^{1} \mathrm{H}-\mathrm{NM} R$ and ${ }^{13} \mathrm{C}$-NM R data see tables 1 and 2 .

Synthesis of 3. To a suspension of $\mathrm{LiAlH}_{4}(1.0 \mathrm{mmol})$ in dry THF (2 ml) was added dropwise $\mathbf{1}(0.1$ $\mathrm{mmol})$ in $\mathrm{THF}(0.5 \mathrm{ml})$ at $0^{\circ} \mathrm{C}$. This mixture was refluxed for $6 \mathrm{~h}$ and left overnight without heating. Acidulated water was added $(20 \mathrm{ml})$ and extracted with $\mathrm{CH}_{2} \mathrm{Cl}_{2}(3 \times 10 \mathrm{ml})$. The organic phase was dried over $\mathrm{CaCl}_{2}$ and evaporated under reduced pressure. The residue was chromatographed on silicagel and elution with $\mathrm{CH}_{2} \mathrm{Cl}_{2} / \mathrm{MeOH} 99: 1$ afforded diol 3 (16.1 mg, 55.3 \%). For ${ }^{1} \mathrm{H}-\mathrm{NMR}$ and ${ }^{13} \mathrm{C}$-NM R data see tables 1 and 2.

Synthesis of 4. Compound $\mathbf{1}(0.1 \mathrm{mmol})$ was added to a mixture of $\mathrm{Ac}_{2} \mathrm{O}(31.7 \mathrm{mmol})$ and pyridine $(37.7 \mathrm{mmol})$ and left at room temperature for $24 \mathrm{~h}$. The reaction mixture was poured onto distilled water $(20 \mathrm{ml})$ and partitioned with $\mathrm{CH}_{2} \mathrm{Cl}_{2}(3 \times 8 \mathrm{~mL})$. The organic layer was washed with water, dried over $\mathrm{CaCl}_{2}$ and purified via silicagel column chromatography with $\mathrm{CH}_{2} \mathrm{Cl}_{2} / \mathrm{MeOH}$ to obtain by elution with 99:1 compound 4 (19.0 mg, $45.3 \%$ ). For ${ }^{1} \mathrm{H}-\mathrm{NMR}$ and ${ }^{13} \mathrm{C}$-NM R data see tables 1 and 2.

Synthesis of 5 Compound $1(0.1 \mathrm{mmol})$ and $\mathrm{K}_{2} \mathrm{CO}_{3}(0.2 \mathrm{mmol})$ were added to DMF $(5 \mathrm{ml})$ and stirred at room temperature for $10 \mathrm{~min}$, after which $\mathrm{ICH}_{3}(0.4 \mathrm{mmol})$ was added. After being stirred for another $24 \mathrm{~h}$, the reaction mixture was poured onto water $(20 \mathrm{ml})$ and partitioned with $\mathrm{CH}_{2} \mathrm{Cl}_{2}(3 \times 8 \mathrm{ml})$ and $\mathrm{AcOEt}(3 \times 10 \mathrm{ml})$. Both organic layers together were washed with saturated $\mathrm{NaCl}$, dried over $\mathrm{CaCl}_{2}$ and chromatographed over silicagel with hexane/AcOEt. Elution with 
hexane/ AcOEt 85:15 afforded methyl ester 5 (16.4 mg, 53.2 \%). For ${ }^{1} \mathrm{H}-\mathrm{NM}$ R and ${ }^{13} \mathrm{C}-\mathrm{NM} \mathrm{R}$ data see tables 1 and 2 .

NMR data for semisynthetic derivatives.

Table 1. ${ }^{1} \mathrm{H}-\mathrm{NM}$ R spectroscopic data for compouns 1-5, $\delta$ in ppm, J in $\mathrm{Hz}$.

\begin{tabular}{|c|c|c|c|c|c|}
\hline \multicolumn{6}{|c|}{ Position } \\
\hline & $1^{\mathrm{a}}$ & $2^{a}$ & $3^{b}$ & $4^{a}$ & $5^{b}$ \\
\hline \multirow[t]{2}{*}{1} & $0.91 \mathrm{~m}$ & 0.92 & $0.98 \mathrm{dd}$ & 0.94 & 0.97 os \\
\hline & 1.79 os & 1.80 os & $\begin{array}{l}(4.2,12.9) \\
1.87 \mathrm{~m}\end{array}$ & $1.81 \mathrm{os}$ & $1.85 \mathrm{~m}$ \\
\hline 2 & $1.40 \mathrm{~m}$ & $1.41 \mathrm{~m}$ & $1.47 \mathrm{~m}$ & $1.42 \mathrm{~m}$ & $1.50 \mathrm{os}$ \\
\hline \multirow[t]{2}{*}{3} & 1.11 os & 1.12 os & 1.16 os & 1.13 os & 1.15 os \\
\hline & $1.36 \mathrm{os}$ & 1.37 os & $1.41 \mathrm{os}$ & 1.37 os & $1.40 \mathrm{~m}$ \\
\hline 5 & $1.13 \mathrm{os}$ & 1.12 os & $1.21 \mathrm{~m}$ & 1.14 os & $1.20 \mathrm{~m}$ \\
\hline \multirow[t]{2}{*}{6} & $1.84 \mathrm{~m}$ & 1.83 & $1.91 \mathrm{~m}$ & $1.87 \mathrm{~d}$ & 1.90 os \\
\hline & 1.97 os & 1.98 & $2.05 \mathrm{~m}$ & $2.01 \mathrm{~d}$ & $2.05 \mathrm{~m}$ \\
\hline 7 & $5.62 \mathrm{br} \mathrm{s}$ & $5.74 \mathrm{br} \mathrm{s}$ & $5.75 \mathrm{~m}$ & $5.74 \mathrm{brs}$ & $5.75 \mathrm{t}(2.7)$ \\
\hline 9 & $1.64 \mathrm{br} \mathrm{s}$ & $1.63 \mathrm{brs}$ & $1.78 \mathrm{br} \mathrm{s}$ & $1.67 \mathrm{brs}$ & 1.77 br s \\
\hline \multirow[t]{2}{*}{11} & 1.09 os & $1.10 \mathrm{os}$ & 1.14 os & $1.12 \mathrm{os}$ & 1.16 os \\
\hline & 1.39 os & 1.38 os & $1.52 \mathrm{~m}$ & $1.39 \mathrm{os}$ & 1.51 os \\
\hline \multirow[t]{2}{*}{12} & 1.09 os & 1.10 os & 1.14 os & 1.11 os & 1.16 os \\
\hline & $1.52 \mathrm{~m}$ & $1.60 \mathrm{os}$ & $1.66 \mathrm{~m}$ & $1.39 \mathrm{os}$ & $1.63 \mathrm{~m}$ \\
\hline 13 & 1.79 os & 1.77 os & 1.57 os & 1.77 os & 1.93 OS \\
\hline \multirow[t]{2}{*}{14} & 1.93 os & 1.91 os & 1.43 os & 1.94 os & $2.16 \mathrm{dd}(7.5)$ \\
\hline & $\begin{array}{l}2.21 \mathrm{dd} \\
(5.4,15.0)\end{array}$ & $\begin{array}{l}2.30 \mathrm{dd} \\
(4.5,14.7)\end{array}$ & 1.57 os & $\begin{array}{l}2.19 \mathrm{dd} \\
(5.7,14.7)\end{array}$ & $\begin{array}{l}2.33 \mathrm{dd} \\
(6.6,14.7)\end{array}$ \\
\hline 15 & & & $3.69 \mathrm{~m}$ & & \\
\hline 16 & $0.87 d(6.5)$ & $0.87 d(6.3)$ & $0.93 d(6.3)$ & $0.87 d(6.6)$ & $0.96 d(6.6)$ \\
\hline \multirow[t]{2}{*}{17} & $3.78 d$ & $4.02 \mathrm{~d}(11.1)$ & $3.97 \mathrm{~d}(12.3)$. & $4.33 d(12.3)$ & $3.96 \mathrm{~d}(12.3)$ \\
\hline & $3.85 d$ & $4.17 \mathrm{~d}(11.1)$ & $4.13 \mathrm{~d}(12.3)$ & $4.48 \mathrm{~d}(12.3)$ & $4.16 \mathrm{~d}(12.3)$ \\
\hline 18 & $0.82 \mathrm{~s}$ & $0.83 \mathrm{~s}$ & $0.86 \mathrm{~s}$ & $0.83 \mathrm{~s}$ & $0.85 \mathrm{~s}$ \\
\hline 19 & $0.84 \mathrm{~s}$ & $0.85 \mathrm{~s}$ & $0.89 \mathrm{~s}$ & $0.84 \mathrm{~s}$ & $0.88 \mathrm{~s}$ \\
\hline \multirow[t]{2}{*}{20} & $0.69 \mathrm{~s}$ & $0.70 \mathrm{~s}$ & $0.75 \mathrm{~s}$ & $0.71 \mathrm{~s}$ & $0.74 \mathrm{~s}$ \\
\hline & & & & $\begin{array}{l}1.99 \mathrm{~s} \\
\left(\mathrm{COCH}_{3}\right)\end{array}$ & $\begin{array}{l}3.66 \mathrm{~s} \\
\left(\mathrm{COOCH}_{3}\right)\end{array}$ \\
\hline
\end{tabular}

${ }^{a}$ Registered in DM SO- $\mathrm{d}_{6},{ }^{b}$ Registered in $\mathrm{CDCl}_{3}$

Table $2 .{ }^{13} \mathrm{C}-\mathrm{NM}$ R spectroscopic data for compouns 1-5, $\delta$ in ppm.

\begin{tabular}{llllll}
\hline Position & & & & & \\
\hline & $\mathbf{1}^{\mathbf{a}}$ & $\mathbf{2}^{\mathbf{a}}$ & $\mathbf{3}^{\mathbf{b}}$ & $\mathbf{4}^{\mathbf{a}}$ & $\mathbf{5}^{\mathbf{b}}$ \\
$\mathbf{1}$ & $38.8 \mathrm{t}$ & $38.7 \mathrm{t}$ & $39.3 \mathrm{t}$ & $38.4 \mathrm{t}$ & $39.2 \mathrm{t}$ \\
$\mathbf{2}$ & $18.6 \mathrm{t}$ & $18.4 \mathrm{t}$ & $18.9 \mathrm{t}$ & $18.3 \mathrm{t}$ & $18.9 \mathrm{t}$ \\
$\mathbf{3}$ & $42.1 \mathrm{t}$ & $41.8 \mathrm{t}$ & $42.4 \mathrm{t}$ & $41.7 \mathrm{t}$ & $42.4 \mathrm{t}$ \\
\hline
\end{tabular}




\begin{tabular}{llllll}
\hline $\mathbf{4}$ & $32.8 \mathrm{~s}$ & $32.6 \mathrm{~s}$ & $33.1 \mathrm{~s}$ & $32.7 \mathrm{~s}$ & $33.1 \mathrm{~s}$ \\
$\mathbf{5}$ & $49.9 \mathrm{~d}$ & $49.5 \mathrm{~d}$ & $50.1 \mathrm{~d}$ & $49.3 \mathrm{~d}$ & $50.0 \mathrm{~d}$ \\
$\mathbf{6}$ & $23.2 \mathrm{t}$ & $23.2 \mathrm{t}$ & $23.9 \mathrm{t}$ & $23.2 \mathrm{t}$ & $23.8 \mathrm{t}$ \\
$\mathbf{7}$ & $121.7 \mathrm{~d}$ & $125.9 \mathrm{~d}$ & $125.7 \mathrm{~d}$ & $127.8 \mathrm{~d}$ & $125.7 \mathrm{~d}$ \\
$\mathbf{8}$ & $139.5 \mathrm{~s}$ & $135.4 \mathrm{~s}$ & $139.7 \mathrm{~s}$ & $134.0 \mathrm{~s}$ & $139.4 \mathrm{~s}$ \\
$\mathbf{9}$ & $52.6 \mathrm{~d}$ & $52.1 \mathrm{~d}$ & $52.9 \mathrm{~d}$ & $52.1 \mathrm{~d}$ & $52.6 \mathrm{~d}$ \\
$\mathbf{1 0}$ & $36.6 \mathrm{~s}$ & $36.4 \mathrm{~s}$ & $36.9 \mathrm{~s}$ & $36.4 \mathrm{~s}$ & $36.9 \mathrm{~s}$ \\
$\mathbf{1 1}$ & $23.8 \mathrm{t}$ & $23.4 \mathrm{t}$ & $24.4 \mathrm{t}$ & $23.3 \mathrm{t}$ & $24.4 \mathrm{t}$ \\
$\mathbf{1 2}$ & $38.6 \mathrm{t}$ & $37.7 \mathrm{t}$ & $38.9 \mathrm{t}$ & $38.0 \mathrm{t}$ & $38.6 \mathrm{t}$ \\
$\mathbf{1 3}$ & $30.8 \mathrm{~d}$ & $30.6 \mathrm{~d}$ & $30.8 \mathrm{~d}$ & $30.4 \mathrm{~d}$ & $31.5 \mathrm{~d}$ \\
$\mathbf{1 4}$ & $41.3 \mathrm{t}$ & $40.9 \mathrm{t}$ & $39.7 \mathrm{t}$ & $41.2 \mathrm{t}$ & $41.4 \mathrm{t}$ \\
$\mathbf{1 5}$ & $174.4 \mathrm{~s}$ & $174.3 \mathrm{~s}$ & $61.3 \mathrm{t}$ & $174.1 \mathrm{~s}$ & $174.1 \mathrm{~s}$ \\
$\mathbf{1 6}$ & $20.0 \mathrm{c}$ & $19.7 \mathrm{c}$ & $20.0 \mathrm{c}$ & $19.8 \mathrm{c}$ & $20.1 \mathrm{c}$ \\
$\mathbf{1 7}$ & $63.4 \mathrm{t}$ & $68.5 \mathrm{t}$ & $66.2 \mathrm{t}$ & $66.9 \mathrm{t}$ & $66.0 \mathrm{t}$ \\
$\mathbf{1 8}$ & $33.2 \mathrm{c}$ & $33.0 \mathrm{c}$ & $33.3 \mathrm{c}$ & $32.9 \mathrm{c}$ & $33.3 \mathrm{c}$ \\
$\mathbf{1 9}$ & $21.9 \mathrm{c}$ & $21.8 \mathrm{c}$ & $22.0 \mathrm{c}$ & $21.7 \mathrm{c}$ & $22.0 \mathrm{c}$ \\
$\mathbf{2 0}$ & $13.7 \mathrm{c}$ & $13.6 \mathrm{c}$ & $13.8 \mathrm{c}$ & $13.5 \mathrm{c}$ & $13.7 \mathrm{c}$ \\
& & & & $170.2 \mathrm{~s}$ & $51.6 \mathrm{c}$ \\
& & & & $20.8 \mathrm{c}\left(\mathrm{COCH}_{3}\right)$ & $\left(\mathrm{COOCH}_{3}\right)$ \\
\hline
\end{tabular}

${ }^{\mathrm{a}}$ Registered in DM SO- $\mathrm{d}_{6},{ }^{\mathrm{b}}$ Registered in $\mathrm{CDCl}_{3}$

\section{Cholinesterase inhibition assay}

Electric eel (Torpedo californica) AChE and horse serum BuChE were used as source of both the cholinesterases. AChE and BuChE inhibitory activities were measured in vitro by the spectrophotometric method developed by Ellman with slight modification ${ }^{7}$. The lyophilized enzyme, 500U AChE/300U BuChE, was prepared in buffer A (8 mM K2 $\mathrm{HPO}_{4}, 2.3 \mathrm{mM} \mathrm{NaH}_{2} \mathrm{PO}_{4}$ ) to obtain $5 / 3 \mathrm{U} / \mathrm{mL}$ stock solution. Further enzyme dilution was carried out with buffer $B$ (8 $\mathrm{mM}$ $\mathrm{K}_{2} \mathrm{HPO}_{4}, 2.3 \mathrm{mM} \mathrm{NaH} \mathrm{PO}_{4}, 0.15 \mathrm{M} \mathrm{NaCl}, 0.05 \%$ Tween $20, \mathrm{pH} 7.6$ ) to produce $0.126 / 0.06 \mathrm{U} / \mathrm{mL}$ enzyme solution. Samples were dissolved in buffer B using $2.5 \%$ of $\mathrm{MeOH}$ as cosolvent. Enzyme solution $(300 \mu \mathrm{L})$ and $300 \mu \mathrm{L}$ of sample solution were mixed in a test tube and incubated for $60 / 120 \mathrm{~min}$ at room temperature. The reaction was started by adding $600 \mu \mathrm{L}$ of the substrate solution (0.5 mM DTNB, $0.6 \mathrm{mM} \mathrm{ATCl} / \mathrm{BTCl}, 0.1 \mathrm{M} \mathrm{Na}_{2} \mathrm{HPO}_{4}, \mathrm{pH} 7.5$ ). The absorbance was read at $405 \mathrm{~nm}$ for $180 \mathrm{~s}$ at $27^{\circ} \mathrm{C}$. Enzyme activity was calculated by comparing reaction rates for the sample to the blank. All the reactions were performed in triplicate. $\mathrm{IC}_{50}$ values were determined with GraphPad Prism 5. Tacrine (99\%) was used as the reference AChE/ BuChE inhibitor.

\section{Results and discussion}

In the present work four derivatives of 17-hydroxycativic acid isolated from $\mathrm{G}$. ventanensis were prepared as depicted in Scheme. Compound $\mathbf{2}$ was obtained by sulfation with trimethylaminesulfur trioxide complex of the hydroxyl group; reduction of the carboxylic acid to alcohol using $\mathrm{LiAlH}_{4}$ afforded diol 3; acetylation with $\mathrm{AC}_{2} \mathrm{O}$ / pyridine of the hydroxyl group gave 4 ; using $\mathrm{K}_{2} \mathrm{CO}_{3}$ and $\mathrm{CH}_{3} \mathrm{l}$, compound $\mathbf{5}$ was synthesized. These derivatives are new diterpenes and have been fully characterized by mono- and bidimensional NM R spectroscopy (Tables 1 and 2). 


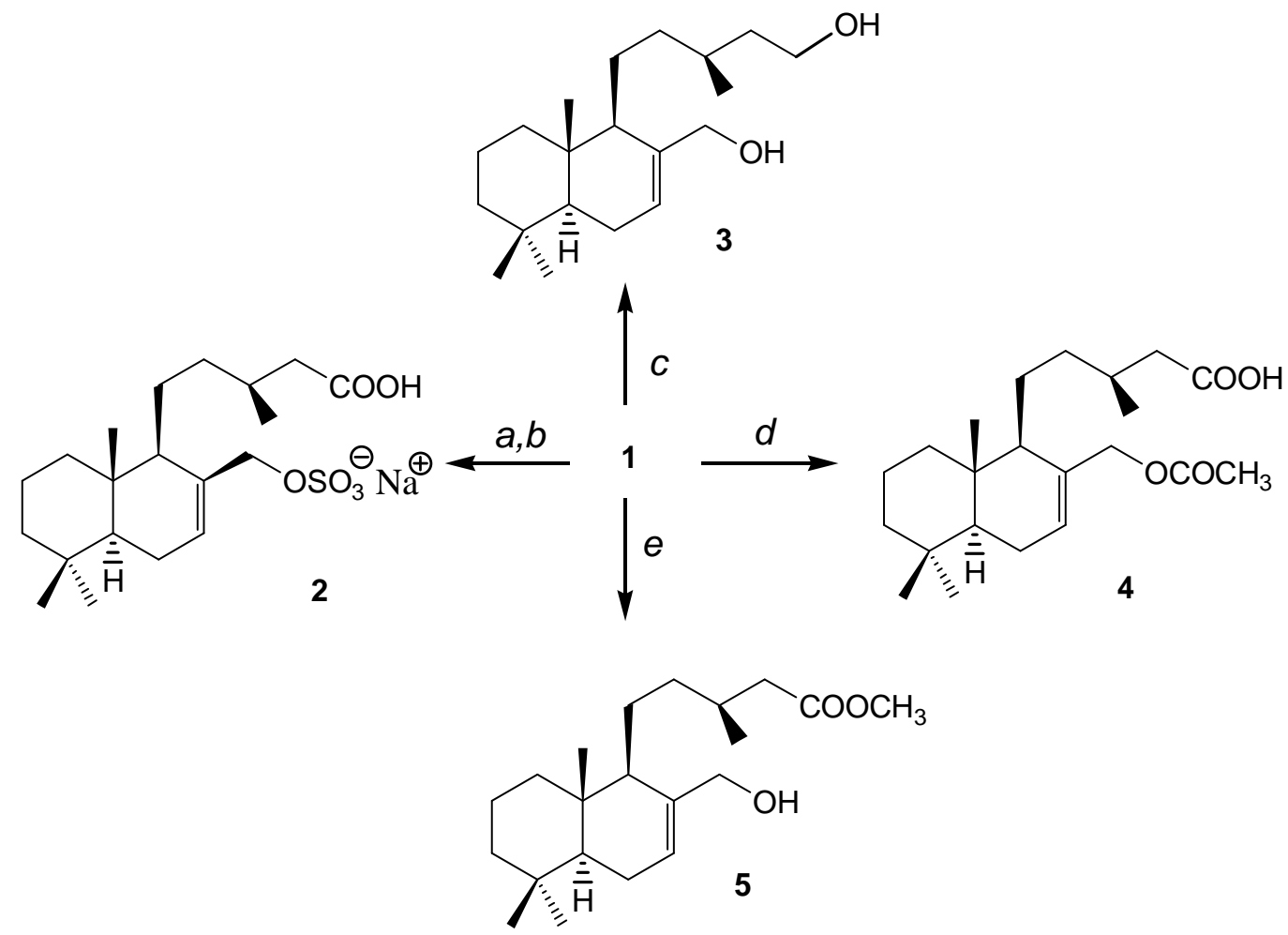

Scheme. (a) $\left(\mathrm{CH}_{3}\right)_{3} \mathrm{~N}_{\mathrm{SO}}$, DM F, 5 min, $150^{\circ} \mathrm{C}$, microwave; (b) Amberlite CG-120 (MeOH); (c) LiAlH4, THF; (d) $\mathrm{AC}_{2} \mathrm{O}$, Py; (e) $\mathrm{ICH}_{3}, \mathrm{~K}_{2} \mathrm{CO}_{3}$, DMF.

The AChE and BuChE inhibitory activity of the semisynthetic compounds $\mathbf{2 - 5}$ were evaluated and compared to that of the natural compound $\mathbf{1}$. Even though $\mathbf{1}$ has a significant inhibition of $\mathrm{AChE}$ $\left(\mathrm{IC}_{50}=21.1 \mu \mathrm{M}\right)$, derivatives $\mathbf{2 - 5}$ showed no AChE inhibitory activity. On the other hand, the BuChE inhibition of 3-5 resulted to be of the same order of magnitude of $\mathbf{1}\left(\mathrm{IC}_{50}=171.1 \mu \mathrm{M}\right)$ with $\mathrm{IC}_{50}$ values 373.2, 219.6 and $143.7 \mu \mathrm{M}$ respectively, showing weak BuChE inhibitory activity. Compound 2 did not inhibit this enzyme.

\section{Conclusions}

These preliminary results suggest that both carboxylic group (C15) and allylic alcohol (C17) could be important functional groups for AChE inhibitory activity of this type of labdane diterpenes. Structural modifications achieved until now have not enhanced AChE inhibition, while compounds 3-5 showed selectivity for BuChE over AChE.

Acknowledgments - This work was financially supported by the National Research Council of Argentina (CONICET), Universidad Nacional del Sur (Argentina) and ANPCYT. A.P.M. is a Research M ember of CONICET. N.P.A. is grateful to CONICET for her doctoral fellowship.

\section{References:}

1- Wang, B.; M ai, Y.-C.; Li, Y.; Hou, J.-Q.; Huang, S.-L-.; Ou, T.-M.; Tan, J.-H.; An, L.-K.; Li, D.; Gu, L.Q.; Huang, Z.-S. Eur. J. Med. Chem. 2010, 45, 1415.

2- Chinou, I. Curr. M ed. Chem. 2005, 12 (11), 1295. 
3- Pertino, M.W.; Theoduloz, C.; Palenzuela, J.A.; Afonso, M.M.; Yesilada, E.; Monsalve, F.; González, P.; Droguett, P.; Schmeda-Hirschmann, G. M olecules 2011, 16(10), 8614.

4- Pertino, M.W.; Theoduloz, C.; Bastías, M.; Schmeda-Hirschmann, G. Molecules 2013, 18, 5936.

5- Schmeda-Hirschmann, G; Pertino, M.W.; Rodríguez, J.A.; Monsalve, F.; Droguett, D.; Theoduloz, C. Molecules 2010, 15, 7378.

6- Schmeda-Hirschmann, G.; Rodríguez, J.A.; Theoduloz, C.; Valderrama, J.A. Planta M ed. 2011, 77, 340.

7- Di Giovanni, S.; Borloz, A.; Urbain, A.; M arston, A.; Hostettmann, K.; Carrupt, P.A.; Reist, M. Eur. J. Pharm. Sci. 2008, 33, 109. 